\title{
Study of Intelligent Integrated Modeling and Development of Agricultural Post-Project Evaluation*
}

\author{
Chen Li \\ Department of Management Engineering, Anhui Institute of Architectural and Industry Hefei, \\ China \\ Chinalichina@163.com
}

\begin{abstract}
Post-evaluation for agricultural project (PEAP) is an important indispensable aspect of agricultural project cycle management. Based on the comparison of the virtual situation and in advanced anticipation of the investigation, PEAP can be used to determine the variation and find the improving method through analyzing the reason, feedbacking the information, and summarizing the lessons, so as to bring economic benefit as much as possible. The post evaluation, especially intelligent integrated agricultural post-project evaluation, started relatively late in our country and has no its own theoretical system. Now days, modeling and development of agricultural post-project evaluation can not fulfill the requirement of social development. The study of intelligent integrated modeling and development of agricultural post-project pvaluation has the important realistic meaning and theoretical value.
\end{abstract}

Keywords: Post-evaluation, Agricultural project, Intelligent integrated.

\section{Introduction}

China is at a stage of rapid economic development. Agricultural projects are more and more important in social and economic life. Post-evaluation for agricultural project (PEAP) is an important indispensable aspect of agricultural project cycle management. Based on the comparison of the virtual situation and in advanced anticipation of the investigation, PEAP can be used to determine the variation and find the improving method through analyzing the reason, feedbacking the information, and summarizing the lessons, so as to bring economic benefit as much as possible. The post evaluation, especially PEAP, started relatively late in our country and has no its own theoretical system but just follows investment project evaluation right now. Thus PEAP can not fulfill the requirement of social development. The PEAP study has the important realistic meaning and theoretical value.

The investment performance of the agriculture project is characterized with instability, regionalism, and dependency. In the past several decades, the contents of

* This research has been funded by the Natural Science Project of Anhui Education Department (Project No.: KJ2009A67). Scientific Research Project, Doctor- Master of aiai (2009) NO.94: Study on the theory and method of post-project evaluation of intelligent integrated modeling. 
PEAP have switched from simple post-evaluation for finance or national economy to broader field including finance, national economy, environment, and society. Meanwhile, the every aspect of evaluation has greatly developed in both depth and width. However, because the PEAP's contents are so complicated and extensive, the restrictions of some factors like concept, regime, and intellectual system have curbed the development of the post-evaluation theory in its research and practice. PEAP has shown some obvious shortages: the faulty contents, the difficulties of choosing assessing index, this limits the application of the post-evaluation for economy, environment and society. There are also many nonlinearity and complex problems in PEAP, which cause it very difficult to establish mathematics' model with accuracy. On the other hand, all information applied in PEAP research has the characters of uncertainty, inaccuracy, and incompleteness. So more systematic and completed theories and methods are required for evaluation. Accordingly, nowadays to introduce Artificial Intelligence (A.I.) would become the main trend in PEAP studies although it is not easy to do so.

\section{The Development of Intelligent Integrated Modeling}

With the rapid of development of computer science, artificial intelligence and microelectronics, people has its own understanding of the mechanism. In 1971, the United States Fu (Fu Jing Sun) proposed the intelligent control concepts based on the combination of theory and artificial intelligence. Intelligent modeling method refers to the industrial process modeling approach which includes expertise in methods, neural networks, fuzzy logic methods, pattern recognition methods. Genetic Programming methods and methods based on genetic algorithms and the most widely used method is the three kinds of intelligent modeling method.

Domestic research shows that intelligent modeling method has been widely used, but it is seldom used in post-project evaluation. CHEN Xiang-gui: Through fuzzy-classified measure, data of permeability modeling on $\mathrm{Pb}-\mathrm{Zn}$ sintering process are classified into low-temperature subspace and high-temperature subspace. Two neural network models with same structure and algorithm are built and integrated. Lu Liang, etc. Research of intelligent integranted autegrated automotive sensor system based on memes. Along with rapid development of the automotive sensors and the farther research of MEMS technology, the automotive sensors based on MEMS have extensive applied foreground. WU MIN., etc: The features of the lead-zinc imperial sintering process include strong nonlinearity, time variance, large time delay, and so on. A soft-sensor model of the burn-through point (BTP) was developed.

\section{Theory of Agricultural Intelligent Integrated Assessment}

Modeling is a scientific method of understanding objective things that based on the long-term practice. As the objective world is a real system, it is complexity and diversity, and the forms of the building model forms are vastly different.

Definition 1: Intelligent integrated modeling refers to two or more methods of the actual process and at least one of these methods to artificial intelligence is neural network, fuzzy logic, expert reasoning and intelligent method of genetic algorithms. The most widely used method is the expert reasoning, neural networks and fuzzy logic methods. 
Expert subjective judgments base on the "Score", "index '," ordinal "," reviews "., and rely on the experience from the experts and knowledge workers. They can deal with qualitative and heuristic knowledge and information. The expert system has strong explanatory function, but there is expert system knowledge acquisition "bottleneck" problem, and the type of precision is low. However, because the traditional evaluation algorithm relies on the expert's knowledge and experiences excessively, the result of the agricultural post-project evaluation is usually less accurate.

Fuzzy comprehensive evaluation is a decision-making process that it is based on the fuzzy environment, and the fuzzy set theory can make a comprehensive quantity evaluation on a system restrained from many uncertain factors. Factors of fuzzy comprehensive evaluation are vague and subjective. AHP fuzzv is an effectiive rnethod, which directly eonstructs the judgment rnatrix in analytic hierarchy process accoording to the fuzzy relative rnernhership degree rnatrix of single evaluation index. In a single-level fuzzy comprehensive evaluation, mistakes often occur when there are excessive evaluation factors. Meanwhile, the overabundant evaluation-set elements will result in difficulties in admeasuring the weights rationally.

Neural network is a characteristic to simulate biological neuron and neural network, through simplifying, summing up and refining a kind of parallel running network summarized. Artificial neural network (ANN) is a rising borderline science. Compared to the mathematical statistics, artificial neural network doesn't need exact mathematical model and it can solve some problems that traditional statistical methods failed to resolve. Artificial neural network (ANN) is a powerful tool for multivariate and nonlinear analysis, and offers an alter-native to traditional statistical methods for optimal monitoring and determination of dynamic systems. It has already become an important method that the data have excavated. But the neural network has its limitation: there are a few defects. For instance, it is slow to disappear, easy to converge to the local extreme point. BP is difficult for the function to get out when it gets into a local extreme point. Too many nodes in the hidden layer will lead to the long time of network learning, even the failure of convergence. An overfit phenomenon exists in the BP network.

Fuzzy comprehensive evaluation depends on experts' opinions strongly, and the weights given by different expert are different slightly. Just like talking above, whether fuzzy comprehensive evaluation or neural network, it can not meet the needs of the agricultural post-project evaluation. Support Vector machine (SVM) is a kind of special small sample theory which is invented by v. n. Vapnik professor who created statistical learning theory SVM is one kind method that has the strict theoretical foundation of computer learning new method, it has already used in pattern recognition and computational intelligence, forecast fields at home and abroad, the extensive attention.

Therefore, if we can combine fuzzy comprehensive evaluation or neural network, SVM with other agricultural post-project evaluation methods, the effect will be far more satisfactory.

\section{The Steps Intelligent Integrated Assessment of Agricultural Post-Project Evaluation}

Intelligent Integrated modeling theory in the agricultural post-project evaluation based on the request of the existing modeling problems in complex agricultural process, 
characteristics. The model will apply the theory of agricultural integrated modeling with the actual agricultural processes and the steps is as followings:

\subsection{Clear Dirty Data and Determine the Modeling of Object Modeling}

There are a number of dirty data in agricultural observation data set derived from agricultural post-project evaluation system. Thus, the data must be carefully and reasonably processed before they are used for agricultural post-project evaluation. Our aim is to establish an intelligent pre-processing algorithm for cleaning to improve convergence and stability of a data filter for agricultural post-project evaluation.

Agricultural integrated modeling is a modern agricultural process control and decision-making, fault diagnosis, system characteristics and effectiveness evaluation of the basis for modeling purposes. Because different models needed to describe the nature of the agricultural process. Agricultural integrated modeling can be determined the corresponding model object.

\subsection{Model Variables}

Model variables include input variables, output variables and intermediate variables that is also called state variables. According to the purpose and object modeling, usually determine the output variables. Then according to some analysis, the factors that affect the output variable, and initially identified the input variables can be understood. Sometimes the relationship between the input variables and output variables is very complex, and we can make use of intermediate variables to refine this complex relationship.

\subsection{The Intelligent Integrated Modeling of Agricultural Post-Project Evaluation to Determine}

Two key factors effected the intelligent integrated modeling of agricultural post-project evaluation. First, the process information that has been collected established model

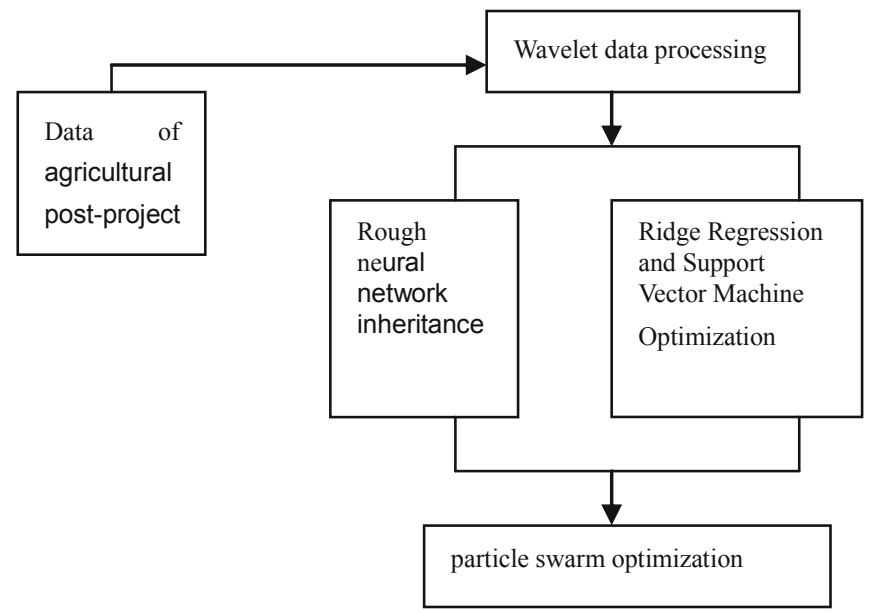

Fig. 1. Model for Integrated form 
primitives. Second, how to integrate the model primitives. The better intelligent integrated modeling of agricultural post-project evaluation is that it can reflect the process mechanism and the model accuracy as high as possible. The methods or fuzzy techniques, neural network, the expert reasoning, and other method are used in the system to analyze the data, and to build the intelligent integrated modeling of agricultural post-project evaluation.

Here, we put forward he SDN (support vector machines dempster neural network) intelligent integrated assessment model. First, we use wavelet analysis of the data to remove noise data. Second, ridge regression, support vector machine, rough neural network , and particle swarm optimization are ensembled, and we conducted a special agricultural post-project.

\subsection{The Model Checking}

This is the final step in modeling of agricultural post-project evaluation. It is used to test whether the model meet the modeling goals and requirements of agricultural post-project evaluation. If you meet the requirements, then that model has been completed; otherwise, you will return to the third step, to further improve the model until a satisfactory model. Through the above steps, get used to the smart integration model of agricultural post-project evaluation.

\section{References}

[1] Amari, S., Wu, S.: Improving support vector machine classifier by modifying kernel functions. Neural Networks 12(9), 783-789 (1999)

[2] Mangasarian, O.L.: Generalized support vector machines. In: Smola, A., Bartlett, P., SchAolkopf, B., Schuurmans, D. (eds.) Advances in Large Margin Classifiers, Cambridge, MA, pp. 135-146 (2000)

[3] Shafer, G.: A Mathematical Theory of Evidence, pp. 2-17. Princeton University Press, Princeton (1976)

[4] Li, Q., Guo, F., Zhou, Y.: A New Satellite Passive Locating Algorithm Using Frequency-only Measurements Based on PSO. Journal of Astronautics 28(6), 1575-1582 (2007)

[5] Parsopoulos, K.E., Vrahatis, M.N.: Particle Swarm Optimizer in Noisy and Continuously Changing Environments. In: Hamza, M.H. (ed.) Artificial Intelligence and Soft Computing, pp. 289-294. IASTED/ACTA Press (2001)

[6] Bakhtazad, A., et al.: Process Trend Analysis using Wavelet based De-noising. Control Engineering Practice, 657-663 (2000) 